\title{
SEIRS model for virus spreading with time delay
}

\author{
Shi-wei Lu ${ }^{1}$, Gang Wang ${ }^{1 *}$, Tong-rui Chen ${ }^{1}$, Run-nian $\mathrm{Ma}^{1}$ \\ ${ }^{1}$ Information and Navigation Institute, Air Force Engineering University, \\ Xi'an, Shaanxi, 710077, China
}

\begin{abstract}
In view of the latent characteristic of virus, the escaped state is considered in the model of virus spreading and employed to describe the state of nodes that are infected but not activated. A novel SusceptibleEscaped-Infected-Removed-Susceptible (SEIRS) model with delay is presented for virus spreading based on the escaped mechanism. In the proposed model, time delay, as an important factor, is considered in the infection stage, as well as the node degree of network. Thereafter, system dynamics equations are derived for the model, and the stable condition of the system is investigated via the criterion of Routh-Hurwitz stability. Finally, simulations are demonstrated to illustrate the proposed model and its performance.
\end{abstract}

\section{INTRODUCTION}

Cyberspace security is a hot issue in the field of person privacy, enterprise interest and government authority [1]. Virus attack, as a means of hackers attacking, has influence on many aspects, such as economy, politics, military and etc. Information diffusion is a hot issue in cyberspace security, which is usually used to study the process of viral diffusion and find its spreading rules.

Most of study in information diffusion model focused on internet society or cyber ecosystem. For instance, Huang et al. 2 established a SIR spreading model where individuals can be infected repeatedly, Zhang et al. [3] investigated two stochastic SIRS models with jumps to describe the spread of network virus by cyber war, terrorism and others, Mustafa 4 used SIQR model to study the identification of mechanisms responsible for recurrent epidemic outbreaks. In addition, considering of the latent character of virus, WANG et.al [5] established SEIQR model to study the spreading rule of virus. Former researches have provided basic model and performance demonstration for information diffusion and virus spreading. However, with the development of virus, more factors should be considered and introduced. The first factor is time delay. Wang and Liu [6, 7] proposed a within-host virus infection model with two delays and studied the effect of time delay on pattern dynamics in a spatial epidemic model. From above we know it needs a while to infect the normal nodes when virus touches with cyber nodes. Consequently, we need to consider the effect of time delay in the process; the second is influence of network degree and elimination rate of nodes. Both of them can affect the stability of the diffusion system. Therefore, in further research, these factors should be taken into consideration on the foundation of previous information diffusion model.
In the paper, a novel information diffusion model is proposed to expound the process of virus spreading. In detail, the novel SEIRS model with time delay is built in section II, and system dynamics equations are established. In section III, stability of the system is concentrated on theoretical research. And numerical simulations are shown in section IV.

\section{SEIRS MODEL}

In general, the node attacked or infected will hide the characteristic of infection and be in a state waiting for activating in the cyber operation. In order to adapt to this case, a new escaped state is brought into to make a further investigation of virus diffusion. In addition, as we know, degree is an important indicator of a network structure. With regard to the topology structure of network, the same virus has the different diffusion speed in the network with different structure [8]. Meanwhile, there is a time slot when virus attacks the normal nodes, such as personal computer or router, and infects them. Therefore, in the process of virus spreading, the time delay of virus infection should be taken into consideration.

A novel information diffusion model with time delay is proposed in Fig.1, based on the traditional and typical SIRS model.

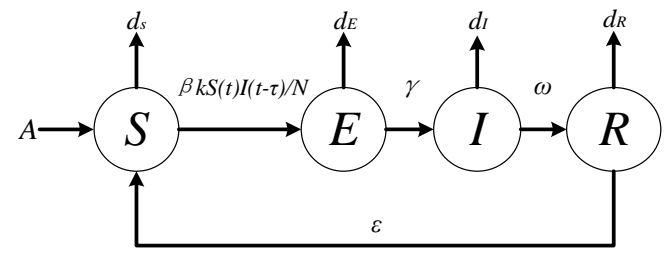

Figure 1. SEIRS model of virus spreading with time delay

\footnotetext{
* Corresponding author:wglxl@nudt.edu.cn
} 
Fig. 1 describes the process of node state transformation, where $A$ denotes the increasing number of susceptible nodes or normal nodes. The elimination rates of susceptible, escaped, infected, and removed nodes are represented by $d_{S}, d_{E}, d_{I}, d_{R}$ respectively. The parameter $\beta$ is the rate of susceptible nodes touched with infected nodes. After a time delay $\tau$, the susceptible nodes will be changed into escaped node. $\beta k S(t-\tau) I(t-\tau) / N$ denotes the number of susceptible nodes infected. $\gamma$ is the rate of virus-latent nodes that will be activated. The infected nodes can be searched and killed by defense system and recover. $\omega$ is the rate of infected nodes recovering healthy state and possessing the ability of antivirus. $\varepsilon$ is the rate of the capability of antivirus decreasing, and $k$ is average degree of network.

Consequently, the corresponding system dynamics equations can be obtained as follows,

$$
\begin{aligned}
\frac{d S(t)}{d t} & =A-\beta k S(t) I(t-\tau) / N-d_{S} S(t)+\varepsilon R(t) \\
\frac{d E(t)}{d t} & =\beta k S(t) I(t-\tau) / N-\left(\gamma+d_{E}\right) E(t) \\
\frac{d I(t)}{d t} & =\gamma E(t)-\left(\omega+d_{l}\right) I(t) \\
\frac{d R(t)}{d t} & =\omega I(t)-\left(\varepsilon+d_{R}\right) R(t)
\end{aligned}
$$

In Eq. (1), $S(\mathrm{t})$ is the number of susceptible nodes at time $t$. And it is similar with the meaning of $E(\mathrm{t}), I(\mathrm{t})$, and $R(\mathrm{t}) . N$ represents the total amount of network nodes.

\section{STABILITY ANALYSIS}

In order to get the balance points of system (1), the differential equations in system (1) are assigned to equal zero. Then balance point can be calculated as

$$
P^{0}\left(S^{0}, E^{0}, I^{0}, R^{0}\right)=\left(\frac{A}{d_{S}}, 0,0,0\right)
$$

There is also another balance point as follows,

$$
\begin{aligned}
& P^{1}\left(S^{1}, E^{1}, I^{1}, R^{1}\right) \\
= & \left(\frac{N\left(\gamma+d_{E}\right)\left(\omega+d_{l}\right)}{\beta k \gamma}, \frac{\left(\omega+d_{l}\right)}{\gamma} I^{1}, I^{1}, \frac{\omega}{\left(\varepsilon+d_{R}\right)} I^{1}\right)
\end{aligned}
$$

Hereby, make the basic reproduction number [9] $R_{0}=A \beta k \gamma / N d_{S}\left(\gamma+d_{E}\right)\left(\omega+d_{I}\right)$. In Eq. (3), let $I^{1}=\left(\varepsilon+d_{R}\right) R_{0} / \beta k\left[\left(\gamma+d_{E}\right)\left(\omega+d_{I}\right)\left(\varepsilon+d_{R}\right)-\varepsilon \gamma \omega\right]$. If and only if $R_{0}<1$, Eq. (1) will have a virus-free equilibrium point $P^{0}$. In contrast, if $R_{0} \geq 1$, it will hold an endemic equilibrium point $P^{1}$. Jacobian matrix at $P^{*}$ is given by $\mathbf{J}\left(p^{*}\right)=\left[\begin{array}{cccc}\frac{-\beta k}{N} I(t-\tau)-d_{s} & 0 & \frac{-\beta k}{N} S(t) & \varepsilon \\ \frac{\beta k}{N} I(t-\tau) & -\gamma-d_{E} & \frac{\beta k}{N} S(t) & 0 \\ 0 & \gamma & -\omega-d_{t} & 0 \\ 0 & 0 & \omega & -\varepsilon-d_{k}\end{array}\right]$

Theorem 1 If $R_{0}<1$, the system is globally asymptotically stable at the disease-free equilibrium point $P^{0}$; Else if $R_{0} \geq 1$, it is unstable at disease-free equilibrium point $P^{0}$.
Proof According to the matrix (4), Jacobian matrix of disease-free equilibrium point $P^{0}$ can be obtained as follow

$$
\mathbf{J}\left(p^{0}\right)=\left[\begin{array}{cccc}
-d_{S} & 0 & \frac{-A \beta k}{N d_{S}} & \varepsilon \\
0 & -\gamma-d_{E} & \frac{A \beta k}{N d_{S}} & 0 \\
0 & \gamma & -\omega-d_{I} & 0 \\
0 & 0 & \omega & -\varepsilon-d_{R}
\end{array}\right]
$$

Solve the eigenvalue polynomial of $J\left(P^{0}\right)$

$$
\begin{aligned}
& \left(\lambda_{1}+d_{S}\right)\left(\lambda_{2}+\varepsilon+d_{R}\right) \\
& \times\left[\left(\lambda_{3}+\gamma+d_{E}\right)\left(\lambda_{4}+\omega+d_{I}\right)-\frac{A \beta k \gamma}{N d_{S}}\right]=0
\end{aligned}
$$

Two characteristic roots of Eq. (6) are respectively $\lambda_{1}=-d_{S}, \lambda_{2}=-\varepsilon-d_{Q}$. If $R_{0}<1$, then $\lambda_{3}, \lambda_{4}$ are less than zero. From Ref. [10], we know disease-free equilibrium point $P^{0}$ is globally asymptotically stable. Furthermore, there is no infected node and the number of other nodes cannot change anymore. When $R_{0} \geq 1$, one of characteristic roots $\lambda_{3}$ or $\lambda_{4}$ must be more than zero. Hence, disease-free equilibrium point $P^{0}$ is unstable.

Theorem 2 If $R_{0} \geq 1$, the system will be locally asymptotically stable at the endemic equilibrium point $P^{1}$

Proof The system (1) can be divided into two parts. $x(t)=(S(t), E(t), I(t), R(t))^{T} \quad$ and the part $x(t-\tau)=(S(t-\tau), E(t-\tau), I(t-\tau), R(t-\tau))^{T} . \quad$ The linear system of system (1) at the equilibrium point $P^{1}$ can be described as follows,

$$
x^{\prime}(t)=B x(t)+D x(t-\tau)
$$

Here, $\mathbf{B}$ and $\mathbf{D}$ are Jacobian matrix of $x(t)$ and $x(t-\tau)$.

$\mathbf{B}=\left[\begin{array}{cccc}-d_{S}-\frac{\beta k}{N} I^{1} & 0 & 0 & \varepsilon \\ \frac{\beta k}{N} I^{1} & -\gamma-d_{E} & 0 & 0 \\ 0 & \gamma & -\omega-d_{I} & 0 \\ 0 & 0 & \omega & -\varepsilon-d_{Q}\end{array}\right]$

$$
\mathbf{D}=\left[\begin{array}{cccc}
0 & 0 & \frac{-\left(\gamma+d_{E}\right)\left(\omega+d_{I}\right)}{\gamma} & 0 \\
0 & 0 & \frac{\left(\gamma+d_{E}\right)\left(\omega+d_{I}\right)}{\gamma} & 0 \\
0 & 0 & 0 & 0 \\
0 & 0 & 0 & 0
\end{array}\right]
$$

Eigenvalue polynomial is following,

$$
\begin{aligned}
& \left(\lambda+\frac{\beta k}{N} I^{1}+d_{S}\right)\left(\lambda+\gamma+d_{E}\right)\left(\lambda+\omega+d_{I}\right)\left(\lambda+\varepsilon+d_{R}\right) \\
& -\varepsilon \gamma \omega\left(\lambda+\frac{\beta k}{N} I^{1}\right)-d_{S} e^{-\lambda \tau}\left(\omega+d_{I}\right)\left(\gamma+d_{E}\right)\left(\lambda+\varepsilon+d_{R}\right)=0
\end{aligned}
$$


and Eq. (10) can be represented as

$$
\lambda^{4}+\mu_{1} \lambda^{3}+\mu_{2} \lambda^{2}+\mu_{3} \lambda+\mu_{4} \lambda e^{-\lambda \tau}+\mu_{5}=0
$$

Here, the coefficients in Eq. (11) are provided as

$$
\begin{aligned}
\mu_{1}= & \frac{\beta k}{N} I^{1}+d_{S}+d_{E}+d_{I}+d_{R}+\varepsilon+\gamma+\omega \\
\mu_{2}= & \left.\frac{\beta k}{N} I^{1}+d_{S}\right)\left(d_{E}+d_{I}+d_{R}+\varepsilon+\gamma+\omega\right) \\
& +\left(\gamma+d_{E}\right)\left(d_{I}+d_{R}+\gamma+\varepsilon\right) \\
& +\left(\omega+d_{I}\right)\left(d_{R}+\varepsilon\right) \\
\mu_{3}= & \left.\frac{\beta k}{N} I^{1}+d_{S}\right)\left[\left(\gamma+d_{E}+\varepsilon+d_{R}\right)\left(\omega+d_{I}\right)+\left(\gamma+d_{E}\right)\left(\varepsilon+d_{R}\right)\right] \\
& +\left(\gamma+d_{E}\right)\left(\omega+d_{I}\right)\left(\varepsilon+d_{R}\right)-\varepsilon \gamma \omega \\
\mu_{4}= & d_{S}\left(\gamma+d_{E}\right)\left(\omega+d_{I}\right) \\
\mu_{5}= & \frac{\beta k}{N} I^{1}\left[\left(\gamma+d_{E}\right)\left(\omega+d_{I}\right)\left(\varepsilon+d_{R}\right)-\varepsilon \gamma \omega\right]
\end{aligned}
$$

When $\tau=0$, the Eq. (11) can be simplified as

$$
\lambda^{4}+\mu_{1} \lambda^{3}+\mu_{2} \lambda^{2}+\left(\mu_{3}+\mu_{4}\right) \lambda+\mu_{5}=0
$$

If $R_{0} \geq 1, \mu_{1}, \mu_{2}>0, \mu_{1} \mu_{2}-\left(\mu_{3}+\mu_{4}\right)>0$, and $\mu_{1} \mu_{2} \mu_{3}-\left(\mu_{3}+\mu_{4}\right)^{2}-\mu_{5}>0$. According to the criterion of Routh-Hurwitz stability, Endemic equilibrium point $P^{1}$ is locally asymptotically stable when $R_{0} \geq 1$.

One way to discuss the stability of $P^{1}$ when $\tau>0$ is to make $\lambda=i w$. The Eq. (11) can be divided into real part and imaginary part,

$$
\begin{gathered}
\omega^{4}-\mu_{2} \omega^{2}-\mu_{4} \omega \sin \omega \tau+\mu_{5}=0 \\
-\mu_{1} \omega^{3}+\mu_{3} \omega+\mu_{4} \omega \cos \omega \tau=0
\end{gathered}
$$

Combine Eq. (13) with Eq. (14), and the new equation about $\omega$ can be get as follows,

$$
\left(\omega^{4}-\mu_{2} \omega^{2}+\mu_{5}\right)^{2}+\left(\mu_{3}-\mu_{1} \omega^{2}\right)^{2}-\mu_{4}^{2}=0
$$

Through calculation, there is no positive real solution of Eq. (15). From Ref. [6], no switch will appear at point $P^{1}$. Therefore, system is locally stable at point $P^{1}$ when $\tau \geq 0$ and $R_{0} \geq 1$.

\section{SIMULATION ANALYSIS}

In this section, the simulations are conducted to investigate the influence of average degree of network and time delay on system stability. With regard to the reproduction number of epidemics $R_{0}=A \beta k \gamma / N d_{S}\left(\gamma+d_{E}\right)\left(\omega+d_{I}\right)$, average degree $k$ of network and time delay $\tau$ are considered to influence the virus spreading of system over time. Thereby, assume that total number of cyber nodes $N=1000$, and the average degree of network $k=10$. In the beginning, there are only susceptible nodes and infected nodes, and their initial value is assigned as $(S(0), E(0), I(0), R(0))=(900,100,0,0)$.

\subsection{THE INFLUENCE OF AVERAGE DEGREE $k$}

Besides the average degree $k$, other parameters can be set as $A=10, \quad \beta=0.2, \quad \gamma=0.6, \omega=0.5, \quad \varepsilon=0.8$, $d_{S}=0.05, d_{E}=0.02, d_{I}=0.1, d_{R}=0.04$. Referring to the method [2,5,6], let the basic reproduction number of epidemics $\quad R_{0}=A \beta k \gamma / d_{S}\left(\gamma+d_{E}\right)\left(\omega+d_{I}\right) N=1$, and then threshold of the average degree $k$ can be get as $k_{\lim }=15.5$. If $k<k_{\lim }$, the system is globally asymptotically stable at the equilibrium point $P^{0}$. Else if $k \geq k_{\text {lim }}$, the system is locally asymptotically stable at the equilibrium point $P^{1}$. Parameters $k=10$ and $k=20$ are assigned, and the result of simulation is shown. The figure shows the number of $S(\mathrm{t}), I(\mathrm{t})$ and $R(\mathrm{t})$. The node $E(\mathrm{t})$ is in scale with $I(\mathrm{t})$.

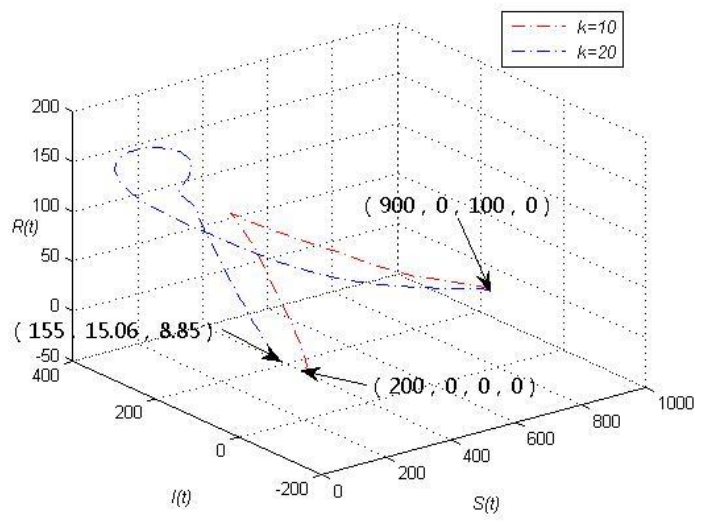

Figure 2. Curve of the number of SIR nodes when $k=10$ and $k=20$

As is drawn in Fig. 2, time interval is set as [0, 500]. The results show that infected nodes approaches to zero when $k=10<k_{\lim }$. That is to say system is globally asymptotically stable at the equilibrium point $P^{0}$ when $R_{0}<1$. In contrast, it is locally asymptotically stable at the point $P^{1}$ when $k=20>k_{\lim }$. Simulations are in good agreement with the theories proof.

The process of virus spreading is described in Fig. 3 and Fig. 4. If the network has a small degree, then the virus can be eliminated rapidly. However, the infected nodes will persist in the system if the degree is large. 


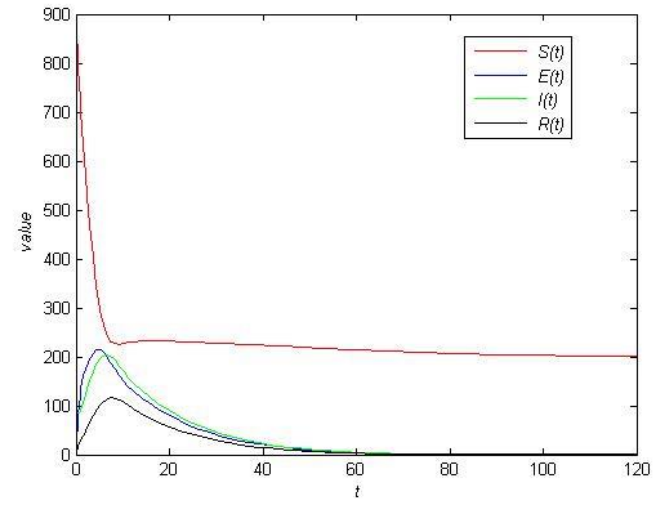

Figure 3. Curve of node number changing with time when $k=10$

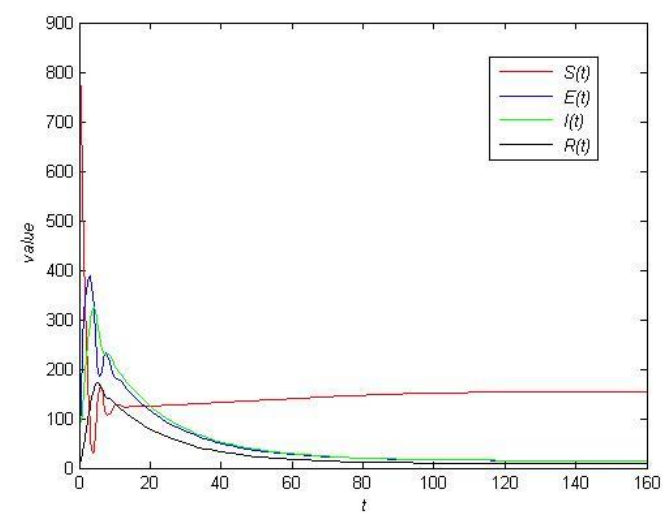

Figure 4. Curve of node number changing with time when $k=20$

\subsection{THE INFLUENCE OF TIME DELAY $\tau$}

The simulation is conducted in the condition of $R_{0}<1$ and $R_{0} \geq 1$. Parameter selection is in correspondence with the section 4.1. The result of simulation is shown as follows.

The curve of the number of nodes is drawn at the Fig. 5 and Fig. 6. The time interval is set as [0,500]. Analysis can be seen that time delay hasn't influence the stability of system. In the different time delay $\tau=1$ and $\tau=10$, system are respectively stable at the equilibrium point $P^{0}$ and $P^{1}$ as well. The results of simulations are in good agreement with the theories proof.

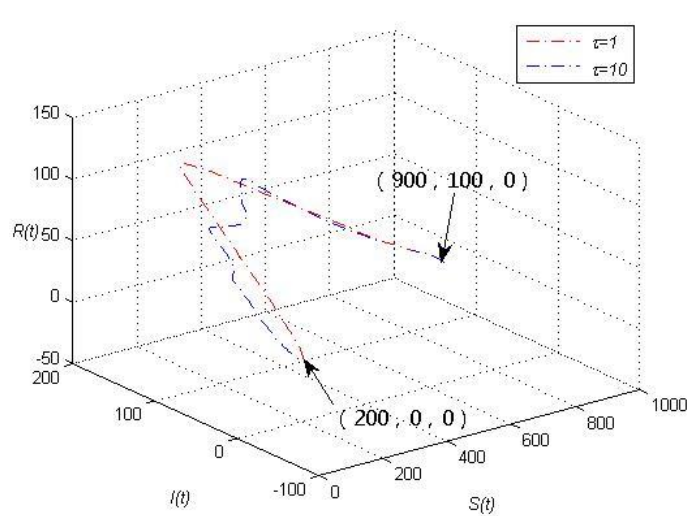

Figure 5. Curve of SIR nodes number under different $\tau$ when $R_{0}<1$

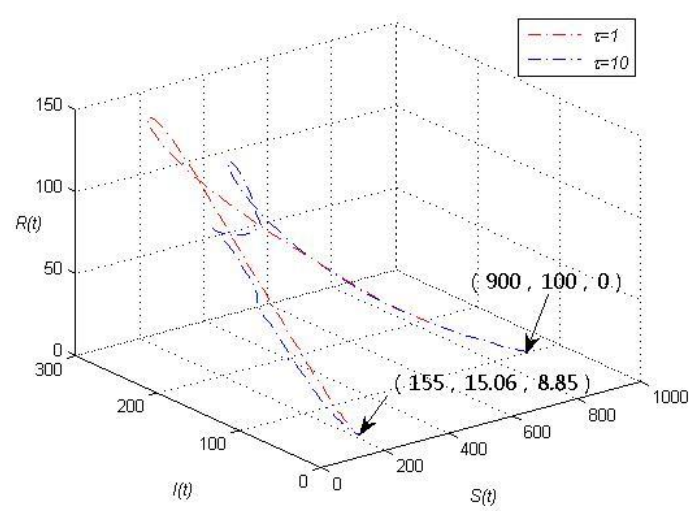

Figure 6. Curve of SIR nodes number under different $\tau$ when $R_{0} \geq 1$

Fig. 7 and Fig. 8 shows the process of virus spreading at the condition of different time delay from where we can see the short delay give a good control of virus diffusion. When $\tau=1$, infected nodes vanish in the interval $[60,70]$. In contrast, it vanished at the interval $[70,80]$ if $\tau=10$. The simulation illustrates that time delay has no influence on system stability. However, it does work for the speed of system tending to the equilibrium solution.

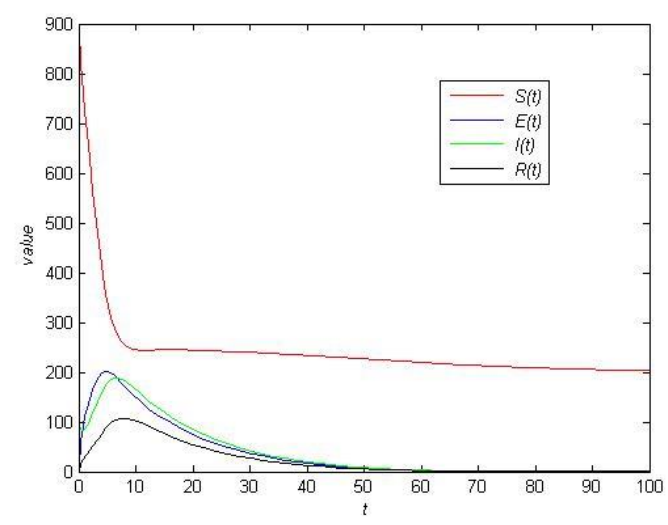

Figure 7. Curve of node number changing with time when $\tau=1$ and $R_{0}<1$ 


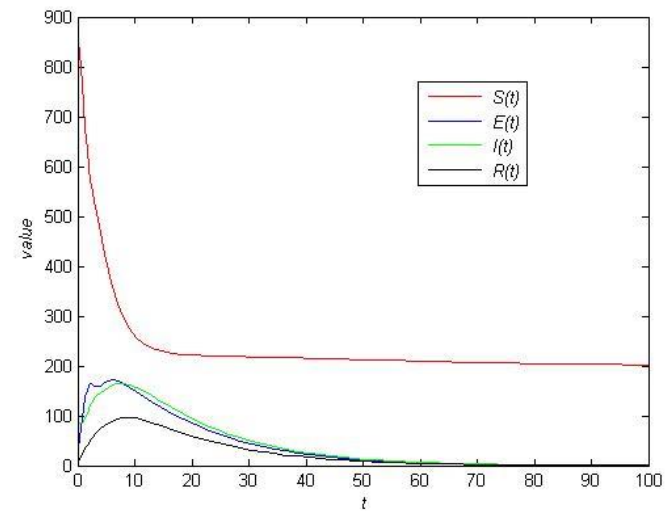

Figure 8. Curve of node number changing with time when

$$
\tau=10 \text { and } R_{0}<1
$$

\section{CONCLUSIONS}

In the paper, a novel virus diffusion model with both escaped state and time delay is investigated. The numerical results are corresponding with the theoretical finding. Specifically, the average degree $k$ and time delay $\tau$ is considered. On the one hand, network degree has the impact on system stability. One way we can obtain to restrain the scope of virus spreading is to reduce the network degree. On the other hand, time delay $\tau$ makes no difference to stability, but it has the effect on the speed of virus spreading. It means that if we can take measure to distinguish virus nodes from network nodes, then virus can be well controlled within a short time. And such is the fact.

\section{ACKNOWLEDGMENT}

The paper is supported by National Natural Science Foundation of China (Program No. 61573017).

\section{REFERENCES}

1. ZEGZHDA D.P, STEPANOVA T.A. Thermodynamic approach to modeling multi-agent systems in cyberspace[J]. Automatic Control and Computer Sciences, 2015, 49(8), 622-626.

2. HUANG Y.H, LI D, FEN Y. A novel epidemic spreading model with decreasing infection rate based on infection times [J]. Physica A: Statistical Mechanics and its Applications, 2016, 444(1), 155165.

3. ZHANG X.H, CHEN F, WANG K. Stochastic SIRS model driven by lévy noise[J]. Acta Mathematica Scientia, 2016, 36(3), 740-752.

4. Mustafa E, Muntaser S, Carlos C. Mathematical Analysis of an SIQR Influenza Model with Imperfect Quarantine[J]. Bulletin of Mathematical Biology, 2017, 79(7), 1612-1636.

5. WANG Z.G, FAN X.M, HAN Q.X. Global stability of deterministic and stochastic multigroup SEIQR models in computer network[J]. Applied Mathematical Modeling, 2013, 37(20-21), 8673-8686.

6. WANG Y, LIU X.N. Stability and Hopf bifurcation of a within-host chikungunya virus infection model with two delays[J]. Mathematics and Computers in Simulation. 2017, 138(1), 31-48.

7. SONG L.P, ZHANG R.P, FENG L.P, et al. Pattern dynamics of a spatial epidemic model with time delay[J], Applied Mathematics and Computation, 2017, 292(1), 390-399.

8. XU D.G, XU X.Y, XIE Y.F. Optimal control of an SIVRS epidemic spreading model with virus variation based on complex networks[J]. Communications in Nonlinear Science and Numerical Simulation, 2017, 48(1), 200-210.

9. YANG Y.M, DAVID G. Proof of conjecture in: The basic reproduction number obtained from Jacobian and next generation matrices-A case study of dengue transmission modeling $[\mathrm{J}]$. Applied Mathematics and Computation, 2015,265(1), 103107.

10. WEI X, WEI J.J. Turing instability and bifurcation analysis in a diffusive bimolecular system with delayed feedback[J]. Communications in Nonlinear Science and Numerical Simulation, 2017, 50(1), 241255. 